\title{
Improving the follow-up of microbiology results: the origins of the Pink Book
}

Henry Boyle, Charlie Hunt, Catherine Mcllroy, Imran Qureshi

Ashford and St Peter's Hospital NHS Trust

\begin{abstract}
Failure to check microbiology results put patients at risk of prolonged infections, resulting in increased morbidity and mortality from sepsis. There are some electronic systems designed to address this risk although they are rarely used in the hospital setting. In many hospitals the follow-up of microbiology results for discharged patients is reliant upon individual doctor's vigilance or ad hoc lists.
\end{abstract}

Our intervention, the 'Pink Book', provides a simple, cost effective system to follow-up microbiology results for discharged patients. This simple paper based system enables prompt, effective, efficient follow-up of microbiology results, saving our paediatric department an estimated 1.5 hours per week, $£ 17,440$ per year and helping to prevent one case of inadequately treated infection per month.

This project highlights how small scale, simple interventions at the local level, born out of the frustration at existing inefficient systems, can make great improvements to patient safety and the efficiency of healthcare.

\section{Problem}

Many children attend A\&E each day with suspected sepsis. They have samples taken which are sent to the microbiology laboratory for culture. Often they are discharged on broad spectrum antibiotics before the results are available. In our district general hospital there was not a robust system to chase outstanding laboratory results, so children with serious infections were not being followed up promptly, leading to a delay in the prescription of potentially life saving antibiotics.

Key problems identified were:

1. A dangerous, unreliable, and haphazard system for the follow-up of laboratory results

2. Missed positive results leading to delay in appropriate antibiotic resulting in avoidable morbidity

3. No assigned responsibility

4. Lack of accountability

This report explains the development and implementation of a simple, cost-effective, novel system to ensure the prompt follow-up of laboratory results for patients no longer in hospital.

\section{Background}

As laboratory tests become more widely available clinicians are requesting more diagnostic tests, dramatically increasing the number of results that need to be checked and acted upon. Gale et al (2011) explored the magnitude of malpractice costs related to communication failures in test result notification.[1] This study examined medical malpractice claims from 52000 American healthcare providers between 2004 to 2008 . They found a total
8.417 malpractice cases citing issues with communication of test results as a major factor in $306(3.6 \%)$ of the cases. Patients did not receive the results in 143 of the 306 cases $(46.7 \%)$ and clinicians did not receive the results in 110 cases $(35.9 \%)$. Other problems included delays in reporting of results $83(27.1 \%)$. The scale of the problem is also increasing. Gale et al estimated that claims payouts as a result of poorly communicated test results rose from $\$ 21.7$ million in 1991 to $\$ 91.0$ million in 2010.

Poor communication of test results impacts upon patient safety across many healthcare systems and is associated with unnecessary mobility, mortality, and increasing malpractice payout costs.

Dumkow et al tested the use of a Culture Follow-Up (CFU) program to improve the follow-up of microbiology cultures for patients discharged from an emergency department [2]. The implementation of a multidisciplinary CFU program was associated with a reduction in emergency department (ED) revisits within 72 hours and hospital admissions within 30 days. They also found that a quarter of patients required post-discharge intervention.

We conducted local research to explore approaches adopted to combat the difficult task of checking outstanding microbiology results for patients who had already been discharged. A number of methods were identified:

1. Personal responsibility: the individual doctor who had requested the test took it upon themselves to chase the outstanding microbiology result

2. Microbiology vigilance: unusual results such as legionella, tuberculosis, or resistant organisms are flagged up by the microbiology team to the doctor who had requested the test. However, this system only worked for unusual organisms 
3. Team responsibility: a number of teams kept a ward or speciality based record of outstanding results. We found two systems in place:

i) A folder was kept in paediatric A\&E with a list of microbiology samples that were sent - these results were then checked by the junior doctor daily and any necessary action was taken

ii) On the general paediatric ward a daily "doctors job book" was kept - the top of each daily job list included outstanding microbiology results to be chased. If the job was not completed then it would be re-written on the following day's job list.

These approaches all partially addressed the issue of chasing microbiology results for discharged patients but none provided a robust, reliable system. As a result, patients were put at increased risk of developing complications from untreated infections.

A literature search of Pubmed revealed no published solutions to this widespread problem. However, there are a number of IT based systems which use an electronic sign off system to ensure every result is seen and acknowledged. Such systems are often employed by GP practices to ensure laboratory results are not missed.

\section{Baseline measurement}

In order to quantify the problem in our hospital, we conducted an audit of the "Doctors Job book" system currently used to chase laboratory results on the general paediatric ward.

General paediatric ward:

The audit measured the time taken to transcribe jobs each day and a record of how many transcribing errors are made over the course of a normal working week.

Results:

Forty-six tasks were transcribed in one week alone, wasting fiftyeight minutes of junior doctors time. More importantly, two errors were made resulting in the microbiology result not being chased and putting patients at risk.

This short audit of just one week on one ward highlighted the inadequacies, inefficiencies, and dangers of the current system. Despite this criticism, it is worth noting that this system was at least attempting to address the issues of missed laboratory results; most wards and departments do not have any measures in place and results are chased in an ad hoc manner.

See supplementary file: ds3641.png - "Graph displaying the slow, inefficient and inaccurate ward based system for recording outstanding microbiology results."
We set about creating a robust, efficient, standardised system for chasing microbiology results in the paediatric department. The aim was to improve patient safety, save time and money, designate responsibility, and facilitate accountability.

We brainstormed a number of ideas and came up with a paper based system, filed in a conspicuous pink ring-binder on each ward which became known as 'The Pink Book of Results'.

The 'Pink Book of Results' is a bright pink folder which contains multiple copies of a proforma. The proforma is split into two parts:

Part $\mathrm{A}$ : is completed by the requesting doctor/nurse and contains prompts to record key patient identifiers, basic clinical details, details of any prescriptions given and any results that are outstanding.

Part B: is for the "chasing" doctor to complete and contains a section to record the result and any action taken, with prompts to remind the chasing doctor to inform the GP and document the results in the notes.

Both parts have clear sections to record date and time of entries, and the signature and contact details of the doctor recording the information.

As the design suggests, blank forms are stored in the pink folder and part $A$ is completed by the requesting doctor. The form then remains in the folder until the result is available and appropriate action is taken. Part B can be completed by the chasing doctor once the result is available and the entire form can be placed in the patients notes as a record of the action taken.

We believe this idea is simple and easy to use, reliable, efficient, sustainable and has already saved time and money. It is:

Simple: the simple proforma is clear and self explanatory. It serves as a reminder as well as documentation of outstanding results and prompts appropriate action.

Cost efficient: for the price of a photocopy and ringbinder this system can prevent delays in diagnoses, prevent morbidity, and ultimately prevent re-admissions and even deaths.

Traceability and accountability: this is not to facilitate a blame culture but is designed to encourage people to think carefully about the investigations they are ordering. It also ensures a point of contact so that further information can be gained from the clinician who saw that patient.

Clinical benefit: legible, clear, detailed record of outstanding results. It removes the need to transcribe jobs and provides all necessary information in a central folder. This reduces the chance that results will be lost to follow up, and ensures that significant results will be checked in a timely fashion.

\section{Design}

\section{Strategy}




\section{BMJ Quality Improvement Reports}

PDSA cycles:

PDSA cycle 1: Design pilot system for A\&E, implement pilot, collect feedback from nurses/doctors, use feedback to amend and implement full scale system into A\&E.

PDSA cycle 2: Implement full scale system into A\&E and conduct tests to compare the impact of our intervention.

PDSA cycle 3: Adapt the A\&E system for the general paediatric ward and special care baby unit (SCBU), implement pilot systems, gather feedback from ward and SCBU nurses/doctors, use feedback to amend and implement full scale system across the paediatric department.

For each cycle, meetings were held with the doctors and nurses of each clinical area to raise awareness and gain feedback from them on what system would be most effective. These meetings provided information and encouraged joint decision making to ensure the new system was effective and well received.

See supplementary file: ds3133.docx - "PDSA Cycles 1, 2 \& 3 demonstrating the evolution of our solution."

\section{Post-measurement}

During PDSA cycle 1 we collected subjective and objective feedback from the staff using the prototype system to highlight areas of strength and weakness, and also assess how useful our proposed system will be.

Our pilot study was received extremely well and the subjective feedback suggested that the new system provided many advantages including:

- Clear and easy to use (100\% agreed or strongly agreed

- Encouraged more detail to be recorded (100\% agreed or strongly agreed)

- Reduced time taken to chase results $(87.5 \%$ agreed or strongly agreed)

- Improved patient safety (100\% agreed or strongly agreed)

- Enabled accountability (100\% agreed or strongly agreed)

- Could be adapted for use on other wards (100\% agreed or strongly agreed)

- Would be better if the folder was brightly coloured eg Pink (62.5\% agreed or strongly agreed)

- There was no consensus on whether the system would be better if it was paper or IT based

(See figure 1 for details)
PDSA cycle 2 revealed that our intervention outperformed the previous system in all 10 areas of quality assessment. Crucial information was recorded more frequently following the implementation of the Pink Book enabling prompt follow-up and action of outstanding microbiology results (See figure 2).

See supplementary file: ds 4123 .docx - "Figures 1 \& 2 showing objective measures of quality improvements and an example of the Pink Book pro forma"

\section{Lessons and limitations}

This project taught us valuable lessons in quality improvement:

- Use daily frustrations as inspiration for quality improvement projects; your project does not need to be ground breaking, small adjustments to make daily tasks easier, more efficient, and safer will save time money and improve patient care.

- Small, simple interventions can make a large difference; by simply redesigning a paper form, raising awareness and changing the colour of the folder we have saved time, money, and ultimately improved patient safety.

- Interventions should be simple and easy to use; this helps overcome the resistance to change.

- Conduct a pilot study and encourage all grades of staff to provide feedback; by involving the whole team in the process we were able to design and implement a system that suited everyones needs.

- Ensure your intervention addresses the problem you have identified.

- Make sure your intervention stands out; by making our intervention bright pink we increased awareness, participation, and increased the effectiveness of our intervention.

Limitations to our project:

- Our intervention for chasing microbiology results remains reliant upon staff completing a paper form, an automated computer system that highlights positive microbiology results would be ideal.

- We were unable to monitor the number of microbiology results that remained unchecked and thus unable to objectively measure the risk posed to patients. An audit of all patients seen over a set time period with close monitoring of how many results remain unchecked would help add weight to this project.

- Alternative IT systems that record which results have been viewed, and highlighted those that have not, would be beneficial for protecting patients and measuring improvements.

\section{Conclusion}

Failure to check microbiology results put patients at risk of 


\section{BMJ Quality Improvement Reports}

prolonged infections resulting in increased morbidity and potential mortality from sepsis. Personal experience, local research, and a literature review found no consistent or documented solution to this common problem.

Our intervention, the Pink Book of Results, provides a simple, cost effective system to follow-up microbiology results for discharged patients. It has been extremely well received by paediatric A\&E staff and is now rolled out across the general paediatric ward and special care baby unit.

The Pink Book enables prompt, effective follow-up of microbiology results saving the paediatric department an estimated 1.5 hours per week, $£ 17,440$ per year, and preventing at least two missed microbiology results per week. It is difficult to quantify the amount of morbidity prevented but anecdotal evidence suggests that our intervention has helped to prevent approximately one case of inadequately treated infection per month.

The most important factors to successful implementation of our quality improvement project were to:

- Involve staff members in the design process

- Ensure the intervention is simple and easy to use, saving them time and effort

- Make the intervention stand out / memorable

- Re-assess regularly to ensure the intervention continues to address the problem at hand

This project highlights how small scale, simple interventions at the local level born out of the frustration at existing inefficient systems can make great improvements to patient safety.

\section{References}

1. Gale BD, Bissett-Siegal DP, Davidson SJ \& Juran DC, Failure to Notify Reportable Test Results: Significance in Medical Malpractice. J Am Coll Rad 2011. 8(11):776-9.

2. Dumkow LE, Kenney RM, Macdonald NC, Carreno JJ, Malhotra M \& Davis SL, Impact of a Multidisciplinary Culture Follow-up Program of Antimicrobial Therapy in the Emergency Department. Infect Dis Ther 2014. 3(1):45-53.

\section{Declaration of interests}

Nothing to declare.

\section{Acknowledgements}

Thank you to:

Dr Cath Mcllroy, Dr Katy Will, Dr Fatima Shurmahi and Mr Charlie Hunt for their hard work in getting this project up and running.
Paediatric staff at St Peter's Hospital, Chertsey, UK, for their help and support in setting up the project.

Dr Imran Qureshi and Dr Keefai Yeong for their advice and guidance in driving the project forward.

Ashford and St Peter's NHS Trust for enabling us to present our project at the International Forum for Quality and Safety, London 2013. 